\title{
Statistical Reconstruction for Quantitative CT Applications
}

\author{
Idris A. Elbakri, Yingying Zhang, Laigao Chen, Neal H Clinthorne and Jeffrey A. Fessler
}

\begin{abstract}
This paper summarizes considerations in developing statistical reconstruction algorithms for polyenergetic X-ray CT. The algorithms are based on Poisson statistics and polyenergetic $X$-ray attenuation physics and object models. In single-kVp scans, object models enable estimates of the contributions of bone and soft tissue at every pixel, based on prior assumptions about the tissue properties. In dual-kVp scans, one can estimate water and bone images independently. Preliminary results with fan-beam data from two cone beam systems show better accuracy for iterative methods over FBP.
\end{abstract}

Index Terms - X-ray computed tomography, dual-energy imaging, penalized-likelihood image reconstruction, tomography, maximum a posteriori (MAP) estimation.

\section{INTRODUCTION}

We are developing a class of statistical algorithms for polyenergetic transmission measurements that account for polyenergetic $\mathrm{X}$-ray sources and attenuation [1]-[5]. The single- $\mathrm{kVp}$ and dual-kVp algorithms significantly reduce beam hardening artifacts, and are promising for quantitative applications.

\section{Models}

The quantity of interest in 2D X-ray CT image reconstruction is the linear attenuation coefficient $\mu(x, y ; \mathcal{E})$, where $(x, y)$ denote spatial coordinates and $\mathcal{E}$ denotes energy. The fundamental challenge is that $\mu$ is a function of three variables whereas a single-kVp sinogram is only two dimensional. Evidently there is a shortage of data.

\section{A. Monoenergetic case}

In the case of a monoenergetic source with energy $\mathcal{E}_{0}$, such as is used in radioisotope transmission scans, e.g., [6], the quantity of interest simplifies to $\mu\left(x, y ; \mathcal{E}_{0}\right)$, In this case reconstruction is feasible, i.e., suffers from only the "usual" amount of illposedness in tomography.

\section{B. Single material case}

If the object consists of materials that all have the same properties in terms of the energy dependence, then we can model the linear attenuation coefficient as follows:

$$
\mu(x, y ; \mathcal{E})=m(\mathcal{E}) \rho(x, y),
$$

Supported in part by NIH grant CA-60711.

I. A. Elbakri is with Fischer Imaging, Denver, CO, L. Chen is with Pfizer BioImaging Center, Ann Arbor, MI, Y. Zhang, N. H. Clinthorne, and J. A. Fessler are with The University of Michigan, Ann Arbor, MI. fessler@umich.edu where $m(\mathcal{E})$ is the (assumed known) mass attenuation coefficient of the material(s), and $\rho(x, y)$ is the unknown density map. For example, in clinical X-ray CT it is common to pretend that the object consists only of soft tissue and that all soft tissue has the spectral properties of water; only the material density varies. Under this assumption, we can reconstruct the density map $\rho(x, y)$ from a single- $\mathrm{kVp}$ sinogram even for a polyenergetic source spectrum using statistical methods, e.g., [1]. However, if the object also contains materials (such as bone) with spectral properties that differ substantially from those of water, then use of the single-material model (1) will lead to undesirable beam hardening artifacts.

\section{Two-material models}

A preferable model for clinical X-ray CT would account for the presence of both water-like and bone-like materials, such as the following:

$$
\mu(x, y ; \mathcal{E})=m_{w}(\mathcal{E}) \rho_{w}(x, y)+m_{b}(\mathcal{E}) \rho_{b}(x, y),
$$

where $m_{w}$ and $m_{b}$ denote the (known) mass attenuation coefficients of water and bone, respectively, and $\rho_{w}$ and $\rho_{b}$ denote the (unknown) densities of water and bone at spatial location $(x, y)$ respectively. Unfortunately, this model has two sets of unknowns, $\left\{\rho_{w}(\cdot)\right\}$ and $\left\{\rho_{b}(\cdot)\right\}$; it seems it would be particularly ill-posed to try to estimate independently both images from a single-kVp scan.

We have explored two options for this situation. The simpler option is to perform a preliminary reconstruction of $\mu$ and then to segment that image into water and bone pixels. In other words, we somehow determine the fractions $f_{w}(x, y)$ and $f_{b}(x, y)$ of water and bone in each voxel. In this case, we can replace (2) with the model

$$
\mu(x, y ; \mathcal{E})=\left[m_{w}(\mathcal{E}) f_{w}(x, y)+m_{b}(\mathcal{E}) f_{b}(x, y)\right] \rho(x, y),
$$

where everything on the right-hand side is known except $\rho(x, y)$. We can reconstruct the density map $\rho(x, y)$ from a polyenergetic single-kVp sinogram using statistical methods [1], [7].

A drawback of that approach is that there can be segmentation errors that will corrupt the estimates of the fractions $f_{w}$ and $f_{b}$, degrading the resulting estimates of the density map $\rho(x, y)$. Our approach to circumvent this problem is to model the fractions $f_{w}$ and $f_{b}$ as functions of $\rho$; functions that we must choose. Generally speaking, for values of $\rho$ less than a bit more than $1 \mathrm{gm} / \mathrm{cc}$, we want the spectral properties to be those 
of water, i.e., $f_{w}=1$ and $f_{b}=0$. When $\rho \gg 1$, we want the spectral properties to be those of bone, i.e., $f_{w}=0$ and $f_{b}=1$. The intermediate values depend on modeling assumptions [2], [4]. The resulting model has the form

$\mu(x, y ; \mathcal{E})=\left[m_{w}(\mathcal{E}) f_{w}(\rho(x, y))+m_{b}(\mathcal{E}) f_{b}(\rho(x, y))\right] \rho(x, y)$,

where $f_{w}(\rho)$ and $f_{b}(\rho)$ must be chosen by the algorithm designer. This approach cannot be perfect in all situations since it only allows one degree of freedom per voxel, but it can produce better results in the single-kVp case than conventional water/bone correction methods [8], [9]. Despite the additional nonlinearities present in the model (3), statistical image reconstruction is feasible [2].

\section{Dual-kVp case}

In the case of dual-kVp (also known as dual-energy) scans, we have two sinograms available, so it becomes feasible to estimate both $\rho_{w}(\cdot)$ and $\rho_{b}(\cdot)$ using statistical methods, e.g., [5].

\section{E. Algorithms}

In all cases, for statistical reconstruction we must discretize the density map(s). Let $\boldsymbol{x}$ denote the coefficients of the discretize map(s). Then one can derive algorithms of the form

$$
\boldsymbol{x}^{(n+1)}=\left[\boldsymbol{x}^{(n)}-\boldsymbol{D}\left(\boldsymbol{x}^{(n)}\right) \nabla \Psi\left(\boldsymbol{x}^{(n)}\right)\right]_{+},
$$

where $\Psi$ is the penalized-likelihood cost function, $\nabla \Psi$ its gradient, and $\boldsymbol{D}$ a diagonal preconditioning matrix. The operator $[\cdot]_{+}$denotes the nonnegativity constraint. One can choose preconditioners that ensure monotone convergence [1], [2], or close approximations thereof. It is straightforward to implement the methods using ordered-subsets acceleration.

\section{DISCUSSION}

To illustrate the use of statistical image reconstruction algorithms for X-ray CT with polyenergetic source modeling, we reconstructed fan beam data from a commercial microCT scanner (GE Genomics and Molecular Imaging) that is used routinely to assess disease progress by quantifying BMD in small animals at Pfizer BioImaging Center. We reconstructed the central fan beam slice from the cone-beam data.

We reconstructed $50 \mathrm{kVp}$ data acquired from scanning a small phantom containing water, air, bone mimic and fat mimic on the microCT scanner. Relative to the FBP method available on the scanner, the statistical approach significantly reduces beam hardening artifacts. The statistical reconstruction estimates water and bone densities within a $5 \%$ error. The fat mimic density is not known, but the error relative to the value of fat density provided on the NIST website is $21 \%$. This is not surprising, since fat is not a mineral-water solution and its spectral attenuation properties deviate somewhat from water and similar soft tissues. Ring artifacts appear in the iterative image. Close examination reveals similar artifacts in the FBP image, obscured by the higher noise level. These artifacts are most likely caused by some detector effect that is not accounted for in the reconstruction.

Preliminary results suggest that the statistical algorithm is more accurate than FBP in quantitatively estimating the density.

To further improve the accuracy of the proposed algorithm, we must estimate the scatter contamination of the data. This may be a relatively minor consideration for animal CT scanners, but it is important for larger objects imaged with large area detectors.

\section{REFERENCES}

[1] I. A. Elbakri and J. A. Fessler, "Statistical image reconstruction for polyenergetic X-ray computed tomography," IEEE Tr. Med. Im., vol. 21, no. 2, pp. 89-99, Feb. 2002.

[2] — "Segmentation-free statistical image reconstruction for polyenergetic X-ray computed tomography with experimental validation," Phys. Med. Biol., vol. 48, no. 15, pp. 2543-78, Aug. 2003.

[3] _ "Efficient and accuract likelihood for iterative image reconstruction in X-ray computed tomography," in Proc. SPIE 5032, Medical Imaging 2003: Image Proc., 2003, pp. 1839-50.

[4] I. A. Elbakri, "Statistical reconstruction algorithms for polyenergetic X-ray computed tomography," Ph.D. dissertation, Univ. of Michigan, Ann Arbor, MI, 48109-2122, Ann Arbor, MI., 2003.

[5] J. A. Fessler, I. Elbakri, P. Sukovic, and N. H. Clinthorne, "Maximumlikelihood dual-energy tomographic image reconstruction," in Proc. SPIE 4684, Medical Imaging 2002: Image Proc., vol. 1, 2002, pp. 38-49.

[6] J. A. Fessler, "Statistical image reconstruction methods for transmission tomography," in Handbook of Medical Imaging, Volume 2. Medical Image Processing and Analysis, M. Sonka and J. M. Fitzpatrick, Eds. Bellingham: SPIE, 2000, pp. 1-70.

[7] B. De Man, J. Nuyts, P. Dupont, G. Marchal, and P. Suetens, "An iterative maximum-likelihood polychromatic algorithm for CT," IEEE Tr. Med. Im., vol. 20, no. 10, pp. 999-1008, Oct. 2001.

[8] P. M. Joseph and R. D. Spital, "A method for correcting bone induced artifacts in computed tomography scanners," J. Comp. Assisted Tomo., vol. 2, pp. 100-8, Jan. 1978.

[9] G. T. Herman and S. S. Trivedi, "A comparative study of two postreconstruction beam hardening correction methods," IEEE Tr. Med. Im., vol. 2, no. 3, pp. 128-35, Sept. 1983. 
microCT data iterative reconstruction

microCT data FBP reconstruction
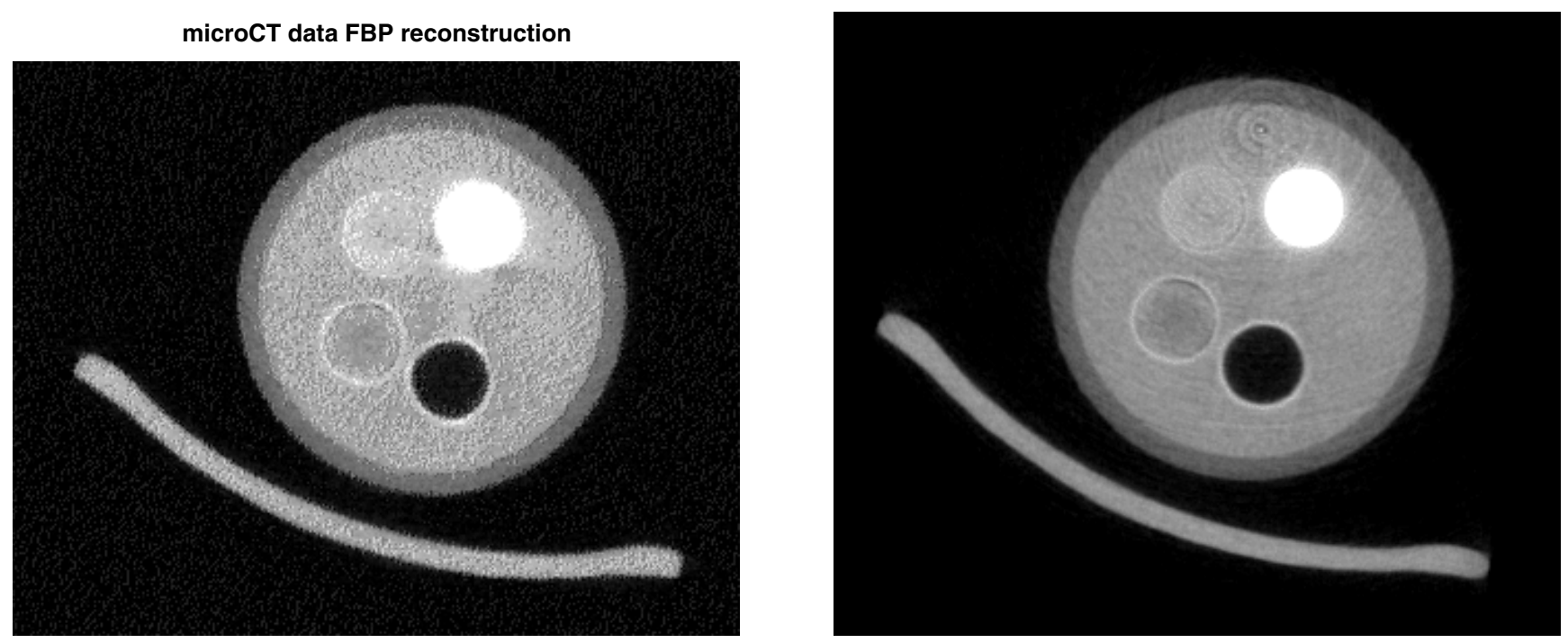

Fig. 1. Phantom reconstruction from microCT. Left: FBP reconstruction. Right: Statistical reconstruction. Window level=-250 HU and width=1500 HU. 